\title{
ESTUDO DO EFEITO DA APROXIMACÃO INICIAL NA CONVERGÊNCIA DO MÉTODO FWI COM DADOS SINTÉTICOS
}

Eliane Santos Cardoso, Universidade Federal do Pará, João Carlos Ribeiro Cruz, Universidade Federal do Pará

Copyright 2019, SBGf - Sociedade Brasileira de Geofísica

This paper was prepared for presentation during the $16^{\text {th }}$ International Congress of the Brazilian Geophysical Society held in Rio de Janeiro, Brazil, 19-22 August 2019.

Contents of this paper were reviewed by the Technical Committee of the $16^{\text {th }}$ International Congress of the Brazilian Geophysical Society and do not necessarily represent any position of the SBGf, its officers or members. Electronic reproduction or storage of any part of this paper for commercial purposes without the written consent of the Brazilian Geophysical Society is prohibited.

\section{Abstract}

This work studies the influence of the initial approximation on the convergence of the FWI (Full Waveform Inversion) method using synthetic data obtained from a three - layer acoustic model whose maximum depth is $1500 \mathrm{~m}$ with variation only in the vertical direction. In the forward and inverse modeling, the IFOS2D code was used, which performs local search by calculating the conjugate gradient of the object function for the estimation of the parameters of the medium. The inversion was performed from four initial models and for each model, the optimization of the object function was evaluated. The density model used remained constant.

\section{Introdução}

A geofísica de exploração marinha tem por objetivo obter uma imagem das estruturas geológicas em subsuperfície. Muitos métodos foram desenvolvidos para isso, dentre os quais, a Inversão da onda completa (FWI) mostrou-se eficiente pra determinar modelos multiparâmetros (velocidade da onda $\mathrm{p}$, velocidade da onda $\mathrm{s}$, densidade) de estruturas complexas em subsuperfície. Esse método se diferencia por utilizar toda a informação contida nos sismogramas observados (fase e amplitude), o que proporciona uma visualização de estruturas de ordem menor que o comprimento da onda sísmica. O método fundamental foi desenvolvido primeiramente pelo $A$. Tarantola, que propôs a teoria do FWI acústico no domínio do tempo (Tarantola, 1986) e outros desenvolvimentos compreendem FWI elástico no domínio do tempo (por exemplo, Tarantola, 1986a, b; Mora, 1987; Crase et al., 1990) e o estabelecimento do FWT no domínio da frequência por RG Pratt (por exemplo, Pratt e Worthington, 1990; Pratt , 1990; Pratt e Goulty, 1991; Pratt, 1999).

O método do FWI tem como objetivo encontrar um modelo de subsuperfície capaz de explicar os dados observados, para isso, um algoritmo de minimização do resíduo entre o dado modelado e o dado observado é desenvolvido iterativamente. A inversão pode ter abordagem elástica ou acústica, dependendo da equação da onda utilizada, sendo que a abordagem elástica requer maior custo computacional devido o aumento no número de parâmetros a serem calculados. O resultado final obtido é diretamente influenciado pela estratégia de inversão utilizada, o que abrange a escolha do operador de precondicionamento do gradiente, a seleção da frequência dominante no dado, e a escolha da função objetivo. Essas diferentes estratégias foram estudadas de forma detalhada em (Przebindowska, Anna, 2013).

O objetivo desse trabalho é aplicar o método FWI através de testes com modelos sintéticos simples para avaliar a dependência da convergência da função objeto em relação ao modelo de velocidade inicial utilizado no algoritmo.

\section{Metodologia}

O FWI é um método de inversão de dados sísmicos que pode utilizar métodos de otimização local e global. Neste trabalho utilizou-se o método de otimização local, ou seja, a partir de uma informação local sobre o gradiente da função objeto, o modelo inicial é melhorado iterativamente. $\mathrm{O}$ código de inversão acústico da forma de onda completa é baseado na abordagem geral de Tarantola (1984) e Mora (1987) formulada no domínio do tempo (Kurzmann, 2012).

A inversão sísmica tem por objetivo encontrar um conjunto de parâmetros do modelo $\mathbf{m}$ capaz de caracterizar adequadamente a subsuperfície da Terra. A validação dos parâmetros encontrados é realizada através do ajuste entre os dados observados, $\mathbf{d}_{o b s}$, e os dados modelados pelo problema direto, $\mathbf{d}_{\text {mod }}$. O desajuste de dados é definido como a diferença entre os dados modelados e os dados observados

$$
\delta \mathbf{d}=\mathbf{d}_{\mathrm{mod}}-\mathbf{d}_{\mathrm{obs}}=\mathrm{f}(\mathbf{m})-\mathbf{d}_{\mathrm{obs}}
$$

Onde $\delta \boldsymbol{d}$ é o vetor desajuste de dimensão $\mathrm{N}$, e $\mathbf{m}$ é o vetor modelo de dimensão M. Para ajustar os dados observados e modelados, a função objeto de norma L2 é minimizada.

$$
E(\mathbf{m})=\frac{1}{2} \delta \mathbf{d}^{T} \delta \mathbf{d}=\frac{1}{2} \sum_{\mathrm{i}=1}^{\mathrm{N}} \delta \mathbf{d}_{\mathrm{i}}^{2}
$$

onde $E(\mathbf{m})$ é a função objeto (função desajuste), e o sobrescrito ${ }^{T}$ denota a transposição da matriz. O somatório é realizado sobre o número de pares fontereceptor e o número de amostras de tempo. 
O método utilizado para minimizar a função objeto e atualizar os parâmetros do modelo é o método do gradiente. O gradiente é dado por

$$
\frac{\partial E}{\partial \mathbf{m}}=\mathbf{J}^{T} \delta \mathbf{d}
$$

Onde $\mathbf{J}^{T}$ é a matriz derivada de Fréchet. Os elementos individuais da matriz são

$$
\mathrm{J}_{i j}=\frac{\partial \mathrm{dmod}_{i}}{\partial \mathrm{m}_{j}}(i=1,2, \ldots, N),(j=1,2, \ldots, M)
$$

A atualização do modelo é realizada através da equação

$$
\mathbf{m}_{n+1}=\mathbf{m}_{n}-\mu_{n}\left(\frac{\partial E}{\partial \mathbf{m}}\right)
$$

\section{A aproximação adjunta}

$\mathrm{Na}$ inversão da forma de onda completa (FWI), o gradiente da função objeto é calculado usando a aproximação adjunta (Mora, 1987;Plessix,2006; Tarantola, 1984). Nessa aproximação, a direção do gradiente é obtido pela correlação cruzada do campo de onda propagado para frente e o campo de onda residual retropropagado.

A relação entre o dado modelado e o modelo pode ser escrita na forma

$$
\mathbf{d}_{\text {mod }}=f(\mathbf{m})=f\left(\mathbf{m}_{0}+\delta \mathbf{m}\right)
$$

Onde $f$ é um operador não-linear que pode ser aproximado utilizando a primeira ordem da expansão da série de Taylor. Uma pequena perturbação no espaço de dados $\delta \mathbf{d}$ que resulta de uma pequena perturbação nos parâmetros do modelo $\delta \mathbf{m}$ pode ser definida como

$\delta \mathbf{d}=\mathrm{f}\left(\mathbf{m}_{\mathbf{0}}+\delta \mathbf{m}\right)-\mathrm{f}\left(\mathbf{m}_{\mathbf{0}}\right)=\boldsymbol{J}_{\mathbf{0}} \delta \mathbf{m}$

Assumindo um modelo de ajuste $\mathbf{m}$ que fornece um bom ajuste entre os dados observados $\mathbf{d}_{\mathrm{obs}} \mathrm{e}$ os dados modelados $\mathbf{d}_{\text {mod }}(\mathbf{m}), \delta \mathbf{d}$ representa os dados residuais através da equação continua (Mora, 1987)

$$
\delta \mathbf{d}(D)=\int_{M} d M \frac{\partial \mathbf{d}(D)}{\partial \mathbf{m}} \delta \mathbf{m}(M)
$$

Onde $M$ e $D$ indicam o espaço do dado e do modelo. Se a matriz derivada de Fréchet for conhecida, é possível integrar as perturbações nos parâmetros do modelo para obter as perturbações no espaço do dado. O gradiente da função objeto (Eq. 3) pode ser escrita na forma contínua

$$
\nabla E_{\mathbf{m}}(M)=\int_{D} d D\left[\frac{\partial \mathbf{d}(D)}{\partial \mathbf{m}}\right]^{*} \delta \mathbf{d}(D)
$$

Onde o sobrescrito $\left(^{*}\right)$ representa adjunto.

\section{Gradiente conjugado}

A abordagem do gradiente conjugado (Mora,1987) é aplicada a fim de melhorar a taxa de convergência do método do gradiente $\delta \boldsymbol{c}_{n}=\delta \mathbf{m}_{n}+\beta_{n} \delta \boldsymbol{c}_{n-1}$

onde $\boldsymbol{c}_{n}$ é o gradiente conjugado, $\delta \boldsymbol{m}_{n}$ é o gradiente de descida mais íngreme e $\beta$ é o escalar que garante que $\delta c_{n}$ e $\delta c_{n-1}$ sejam conjugados. A atualização dos parâmetros do modelo é realizada através da equação

$\mathbf{m}_{n+1}=\mathbf{m}_{n}-\mu_{n} \delta \boldsymbol{c}_{n}$

O gradiente de cada parâmetro do modelo é normalizado para seu valor máximo e $\mu_{n}$ é o comprimento de passo ótimo da iteração n. Para calcular o valor de $\mu_{n}$ (Kurzmann et al., 2009), a função objeto é aproximada pelo ajuste de uma parábola, sendo o seu mínimo correspondente ao comprimento ideal do passo, tal que

$\mu_{n}=\frac{-b}{a}$

Onde a e b são obtidos pela solução de um sistema de três equações.

\section{Resultados}

É apresentada a aplicação do método de inversão da forma de onda completa (FWI) sobre um dado sintético obtido a partir de um modelo acústico de três camadas, cuja profundidade máxima é de $1500 \mathrm{~m}$ com variação apenas na direção vertical. Na modelagem direta e inversa foi utilizado o código IFOS2D (GROOS et al., 2017), que realiza busca local através do cálculo do gradiente conjugado da função objeto para a estimativa dos parâmetros do meio.

Realizamos quatro simulações do FWI com diferentes modelos de velocidade inicial. Os modelos iniciais utilizados foram obtidos pela perturbação de $25 \%(B)$, $35 \%(C)$ e $50 \%(D)$ das velocidades da segunda e terceira camada de um modelo inicial de referência $(A)$. O primeiro teste de inversão utilizou o modelo $A$, enquanto que 0 segundo, terceiro e quarto teste utilizaram os modelos $B$, $\mathrm{C}$ e $\mathrm{D}$, respectivamente. O modelo de densidade utilizado se manteve constante.

A Figura 1 apresenta o resultado do primeiro teste de inversão, em que utilizamos o modelo $A$ como modelo de velocidade inicial. Os perfis de velocidade vertical em $x=500 \mathrm{~m}$ e $\mathrm{x}=750 \mathrm{~m}$ conseguem se ajustar perfeitamente à curva da velocidade inicial (preto tracejado), confirmando a convergência do algoritmo, mas não se aproximou da curva de velocidade verdadeira (cinza), o que pode indicar que o gradiente está preso em um mínimo local que não corresponde ao mínimo global.

As Figuras 2, 3 e 4 apresentam os resultados de inversão para os modelos $B, C$ e $D$, respectivamente. Nos três casos observamos, através dos perfis de velocidade vertical em $x=500 \mathrm{~m}$ e $x=750 \mathrm{~m}$, que o algoritmo converge para um mínimo. Assim como no primeiro teste, não conseguiram se aproximar do modelo real.

A Figura 5 (a) mostra a convergência das quatro curvas da função objeto obtidas pelas simulações do FWI com os respectivos modelos $A, B, C$ e $D$. A Figura $5(b)$ mostra os modelos de velocidade inicial correspondentes às curvas da função objeto. Notou-se que a curva da função 
objeto referente ao modelo de velocidade inicial (azul) apresenta a convergência mais rápida. Enquanto que, as curvas referentes aos modelos de velocidades com aumento progressivo de perturbações na segunda e terceira camada, se afastaram proporcionalmente do caminho de convergência.

A partir da Figura 5 (a), nota-se que o algoritmo consegue alcançar o objetivo de minimizar a função objeto, entretanto, não obteve um modelo invertido próximo do modelo verdadeiro (cinza), o que reforça a presença do problema de não-linearidade na inversão.
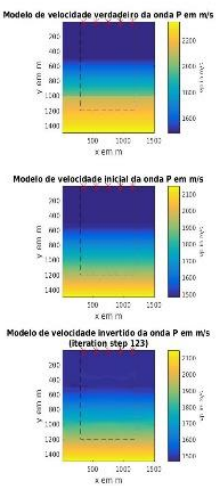
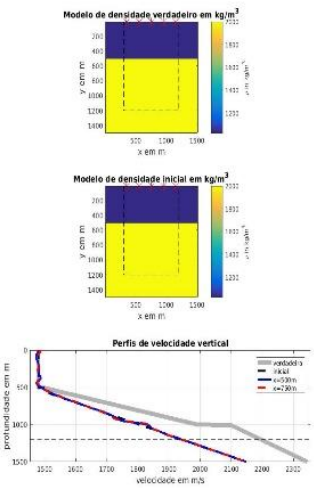

Figura 1: No lado esquerdo estão os modelos de velocidades e no lado direito os modelos de densidades seguido dos perfis de velocidade vertical em $x=500 \mathrm{~m}$ e $x=750 \mathrm{~m}$. O modelo de velocidade inicial corresponde ao modelo de referência. $O$ retângulo tracejado representa a área de inversão dos parâmetros
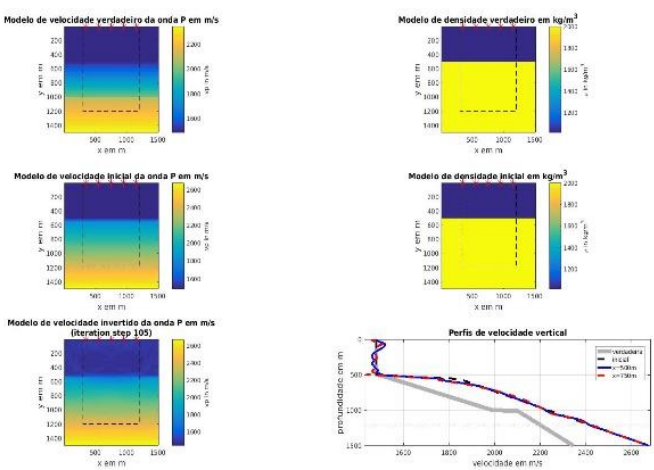

Figura 2: No lado esquerdo estão os modelos de velocidades e no lado direito os modelos de densidades seguido dos perfis de velocidade obtidos. O retângulo tracejado representa a área de inversão dos parâmetros
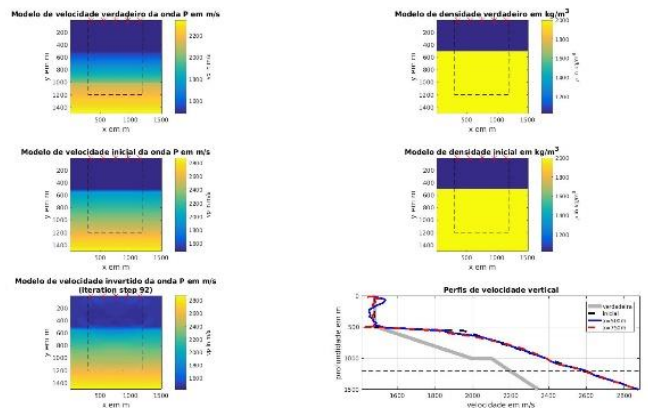

Figura 3: No lado esquerdo estão os modelos de velocidades e no lado direito os modelos de densidades seguido dos perfis de velocidade obtidos. O retângulo tracejado representa a área de inversão dos parâmetros.
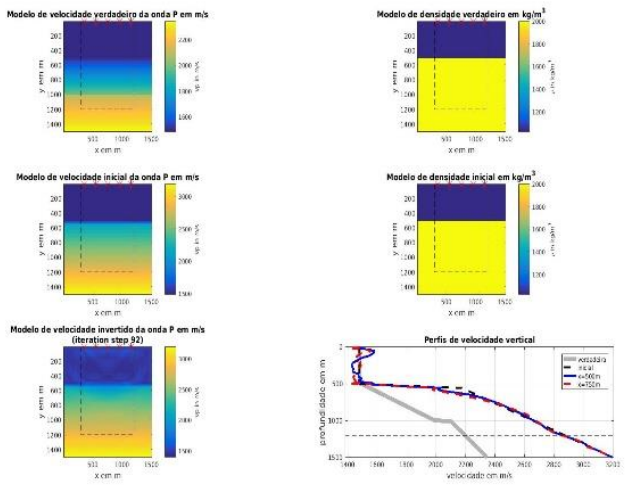

Figura 4: No lado esquerdo estão os modelos de velocidades e no lado direito os modelos de densidades seguido dos perfis de velocidade obtidos. O retângulo tracejado representa a área de inversão dos parâmetros.

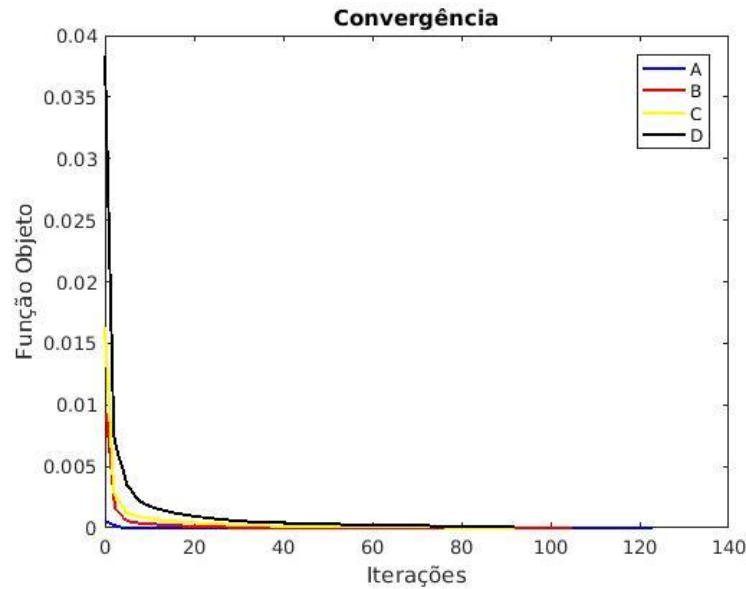

(a) 


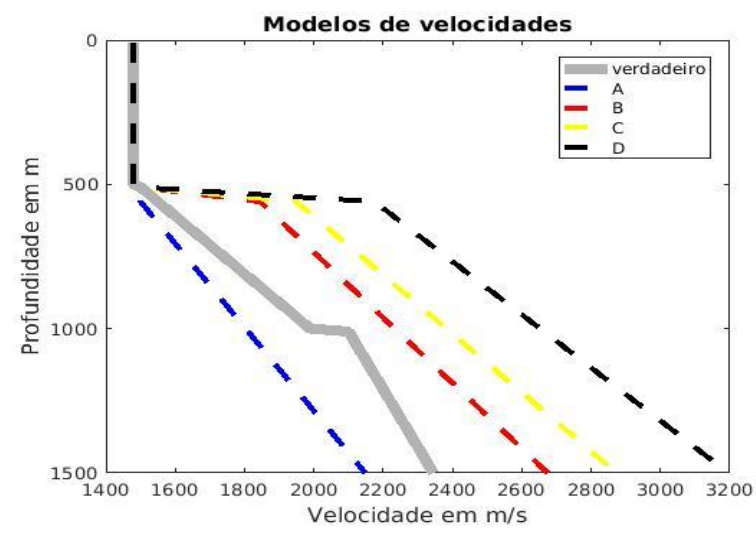

(b)

Figura 5: (a) Gráfico da convergência da função objeto para diferentes modelos de velocidades de partida em função do número de iterações. (b) Modelos de velocidades de partida utilizados em cada simulação do FWI.

\section{Conclusões}

A partir dos resultados apresentados anteriormente, concluímos que o algoritmo consegue alcançar o objetivo de minimizar a função objeto, entretanto, não obteve modelos invertidos próximos do modelo verdadeiro, o que indica que os modelos iniciais utilizados são insatisfatórios para a inversão. Para melhorar os modelos iniciais, é necessário aperfeiçoar a estratégia de inversão utilizada através de estudos sobre a influência do offset $\mathrm{e}$ frequência do dado utilizado, além do precondicionamento do gradiente.

\section{Agradecimentos}

Laboratório de Inversão de Ondas Sísmicas, Universidade Federal do Pará.

Coordenação de Aperfeiçoamento de Pessoal de Nível Superior (CAPES).

\section{Referências}

TARANTOLA, Albert. Inversion of seismic reflection data in the acoustic approximation. Geophysics, v. 49, n. 8, p. 1259-1266, 1984.

TARANTOLA, Albert. A strategy for nonlinear elastic inversion of seismic reflection data. Geophysics, v. 51, n. 10, p. 1893-1903, 1986.

Plessix, R.-E. (2006). A review of the adjoint-state method for computing the gradient of a functional with geophysical applications. Geophysical Journal International, 167:495-503. 7
PRZEBINDOWSKA, Anna. Acoustic full waveform inversion of marine reflection seismic data. 2013. Tese de Doutorado. KIT-Bibliothek.

KURZMANN, André. Applications of 2D and 3D full waveform tomography in acoustic and viscoacoustic complex media. 2012. Tese de Doutorado. Verlag nicht ermittelbar.

Kurzmann, A., Köhn, D., Przebindowska, A., Nguyen, N., \& Bohlen, T. (2009). 2D acoustic full waveform tomography: performance and optimization. In 71st EAGE Conference and Technical Exhibition.

GROOS, Lisa et al. Application of a complete workflow for 2D elastic full-waveform inversion to recorded shallow-seismic Rayleigh waves. Geophysics, v. 82, n. 2, p. R109-R117, 2017.

MORA, Peter. Nonlinear two-dimensional elastic inversion of multioffset seismic data. Geophysics, v. 52, n. 9, p. 1211-1228, 1987.

PRATT, R. Gerhard; WORTHINGTON, M. H. Inverse theory applied to multi-source cross-hole tomography. Part 1: Acoustic wave-equation method. Geophysical prospecting, v. 38, n. 3, p. 287-310, 1990.

PRATT, R. Gerhard; GOULTY, Neil R. Combining wave-equation imaging with traveltime tomography to form high-resolution images from crosshole data. Geophysics, v. 56, n. 2, p. 208-224, 1991. 